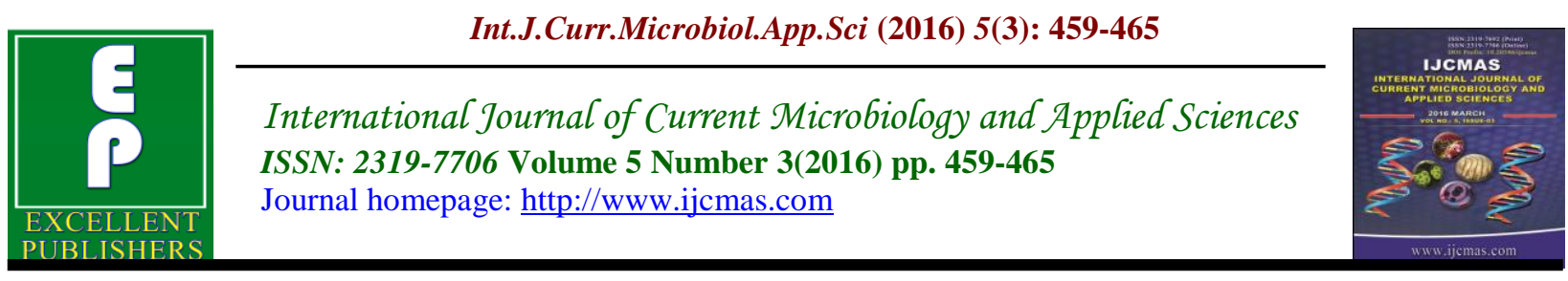

Original Research Article

http://dx.doi.org/10.20546/ijcmas.2016.503.054

\title{
Effect of Meteorological Factor on Fluctuation of Aeromycoflora of Barleria prionitis $\mathbf{L}$.
}

\author{
Diksha Khare* and K.L.Tiwari \\ ${ }^{1}$ Head, Dept of Botany Govt.G.N.A.PG College, Bhatapara, Balodabazar, Chhattisgrah, India \\ ${ }^{2}$ Registrar, Ayush University, Raipur, Chhattisgrah, India \\ *Corresponding author
}

\begin{tabular}{ll}
\hline & A B S T R A C T \\
\cline { 2 - 3 } Keywords & $\begin{array}{l}\text { During the present investigation } 57 \text { fungal species belong to } 27 \text { genera of } \\
\text { fungi were isolated from the aeromycoflora of Barleria prionitis plants. The }\end{array}$ \\
$\begin{array}{l}\text { Barleria prionitis, } \\
\text { Density, } \\
\begin{array}{l}\text { Aeromycoflora, } \\
\text { Meteorological } \\
\text { data. }\end{array}\end{array}$ & $\begin{array}{l}\text { maximum density of the aeromycoflora was observed in winter season and } \\
\text { in the month of November. Minimum density was observed in summer } \\
\text { season and in the month of May. The variations in meteorological factor }\end{array}$ \\
\hline $\begin{array}{l}\text { Article Info } \\
\text { Viz. Temperature, Relative humidity, Rainfall on dispersion of airspore } \\
\text { Accepted: }\end{array}$ & $\begin{array}{l}\text { where further discussed. The linear and non-parametric correlation between } \\
\text { numbilable Online: } \\
\text { 10, March 2016 }\end{array}$ \\
\hline
\end{tabular}

\section{Introduction}

Environmental abiotic factor consist of temperature, relative humidity, rainfall, while and biotic components have actinomycties, bacterial and fungal spore. Fungal spore are major part of air spore. Aerobiological studies are widely used to determine the fungal spectrum in the air. The fungal flora of air is not constant and is highly variable (Anderson 1985). It may originate from different resources in to the air to settle down for growth on suitable host. Fungal spores are an ever-present component of the atmosphere and are present in almost all seasons of the year (Burch and Levetin, 2002; Troutt and
Levetin, 2001). Nevertheless, the meteorological conditions is cause of dispersion of spores and closely related to it variations (Sabariego et al., 2000). The environmental factors affect the daily and seasonal rhythms of airborne fungal and difficult to estimate that are known to importance of each factor (Kasprzyk 2006; Horner et al., 1995). The most effective and reliable application of the relationships between spore production and different environmental growth conditions, can be used for pesticides, or to improve diagnosis and treatment of respiratory allergic diseases (Rodríguez-Rajo et al., 2005). The current 
studies carried out over Barleria prionitis $L$. (Family Acanthaceae), with lots of medical application (Swapna et al., 2011; Purohit and Vyas, 2004).

\section{Materials and Methods}

Barleria prionitis Linn. is a medicinal plant were cultivated in Botanical Garden of Govt. Science college Raipur. During present studies aeromycoflora of above plant was observed fortnightly with the help of gravity petriplate method (Diksha Khare and Tiwari, 2015). The collected spores are identified based on their characteristics such as shape, size and other morphological feature and literature can also be used for authentic identification. The densities were evaluated from following formulae,

Total No. of colonies of individual species Density $=\frac{}{\text { Total No. of observation }}$

Meteorological data [Temperature (Temp), Rainfall (RAF), Relative Humidity (RH)] obtain from the meteorological department of Indra Gandhi Krishi Vishwavidhyala, Raipur Chhattisgarh. The statistical analyses were carried out by SPSS version16.

\section{Results and Discussion}

During one year studies, 778 colonies (57 fungal species) were isolated from the aeromycoflora of Barleria prionitis in different month Table No. 1. Density represent the numerical strength of a species in the community. The number of individual of the species in any unity area is its density. Among 57 fungal species 3 species from Zygomycotina, 3 species from Ascomycotina and 51 species from Anamorphic fungi were recorded. From Table No.2 certain fungal species like Aspergillus niger, A. flavus, A. versicolar, $A$. fumigatus, Alternaria alternata, Curvularia lunata, Cladosporum oxysporum, Fusarium oxysporum were showed maximum density throughout the year. While some fungal species showed minimum density like Rhizopus rhizopodiformis Cunninghamella blackesleeana, Thielavia boothi, Thielavia terricola, Aspergillus nidulens var. latus, Penicillium purpurogenum, Alternaria brassicola, A.humicola, Curvularia eragrostidis, C. ovoidea, C. pallescens, $C$. sengealensis, $C$. lunata var. aeria,

Table.1 Showing Total Number of Colonies of Aeromycoflora of Different Month

\begin{tabular}{llc}
\hline S. No & Month & Total no of colony (TNC) \\
\hline 1 & July & 82 \\
2 & August & 61 \\
3 & September & 55 \\
4 & October & 88 \\
5 & November & 100 \\
6 & December & 87 \\
7 & January & 81 \\
8 & February & 50 \\
9 & March & 48 \\
10 & April & 45 \\
11 & May & 36 \\
12 & June & 45 \\
\hline & Total & $\mathbf{7 7 8}$ \\
\hline
\end{tabular}


Table.2 Showing Densities of Different Fungi in Different Season and

Month of Aeromycoflora

\begin{tabular}{|c|c|c|c|c|c|c|c|c|c|c|c|c|c|c|c|c|c|}
\hline \multirow[t]{2}{*}{ S.No } & \multirow[t]{2}{*}{ NAME OF FUNGI } & \multicolumn{3}{|c|}{ RAINY SEASON } & \multirow[b]{2}{*}{ Oct } & \multirow[b]{2}{*}{ Total } & \multicolumn{5}{|c|}{ WINTER SEASON } & \multicolumn{6}{|c|}{ SUMMER SEASON } \\
\hline & & July & Aug & Sep & & & Nov & Dec & Jan & Feb & Total & Mar & April & May & June & Total & Grand Total \\
\hline & ZYGOMYCOTINA & & & & & & & & & & & & & & & & \\
\hline 1 & Cunninghamella blakesleeana & 0.10 & - & - & - & 0.025 & - & - & - & - & - & - & - & - & - & - & 0.008 \\
\hline 2 & Rhizopus rhizopodiformis & - & - & - & - & - & - & - & 0.10 & & 0.025 & & & 0.1 & - & 0.025 & 0.016 \\
\hline \multirow[t]{2}{*}{3} & Syncephalastrum racemosum & 0.10 & & 0.30 & & 0.10 & 0.10 & - & - & - & 0.25 & 0.3 & - & 0.2 & - & 0.125 & 0.08 \\
\hline & ASCOMYCOTINA & - & - & - & - & - & - & - & - & - & - & - & - & & - & - & - \\
\hline 1 & Chaetomium globosum & - & - & - & - & - & - & - & - & 0.40 & 0.10 & 0.30 & - & & - & 0.075 & 0.058 \\
\hline 2 & Thielavia boothi & - & - & - & - & - & - & - & - & - & - & - & - & 0.10 & & 0.025 & 0.008 \\
\hline \multirow[t]{2}{*}{3} & Thielavia terricola & - & - & - & - & - & - & - & - & - & - & - & - & 0.10 & 0.10 & 0.025 & 0.016 \\
\hline & ANAMORPHIC FUNGI & - & - & - & - & - & - & - & - & - & - & - & - & - & - & - & - \\
\hline 1 & Alternaria alternate & 0.70 & 0.50 & 0.30 & 0.80 & 0.575 & 0.10 & 0.20 & 0.50 & 0.30 & 0.275 & 0.20 & & 0.10 & 0.30 & 0.15 & 0.333 \\
\hline 2 & Alternaria brassicola & - & - & - & - & - & - & 0.30 & 0.20 & 0.20 & 0.175 & - & - & - & - & - & 0.058 \\
\hline 3 & Alternaria chlamydospora & - & - & 0.50 & - & 0.125 & - & 0.40 & 0.20 & 0.10 & 0.175 & - & - & - & - & - & 0.10 \\
\hline 4 & Alternaria citri & 0.60 & 0.30 & 0.30 & 0.40 & 0.40 & 0.10 & 0.20 & - & - & 0.075 & - & - & - & - & - & 0.158 \\
\hline 5 & Alternaria humicola & - & - & - & - & - & - & - & - & - & - & - & - & 0.10 & - & 0.025 & 0.008 \\
\hline 6 & Alternaria radicina & 0.50 & 0.20 & - & - & 0.175 & - & - & 0.3 & - & 0.075 & - & - & - & - & - & 0.08 \\
\hline 7 & Aspergillus nidulans var. latus & - & - & - & - & - & - & - & - & - & & & 0.1 & 0.10 & & 0.05 & 0.016 \\
\hline 8 & Aspergillus nidulans var. acristatus & - & - & - & - & - & - & - & - & 0.10 & 0.025 & 0.10 & 0.2 & 0.10 & & 0.1 & 0.041 \\
\hline 9 & Aspergillus awamori & - & - & - & 0.60 & 0.15 & 0.40 & & & & 0.10 & & & 0.20 & 0.10 & 0.075 & 0.108 \\
\hline 10 & Aspergillus flavus & 0.60 & 0.50 & 0.20 & 0.40 & .425 & 0.30 & 0.50 & 0.60 & 0.40 & 0.45 & 0.50 & 0.7 & 0.80 & 0.60 & 0.65 & 0.508 \\
\hline 11 & Aspergillus fumigates & 0.40 & 0.20 & - & - & 0.15 & 0.60 & 0.40 & & 0.20 & 0.30 & 1.10 & 0.9 & 0.70 & 0.70 & 0.85 & 0.433 \\
\hline 12 & Aspergillus nidulans & - & - & - & - & - & 0.50 & 0.30 & - & - & 0.20 & & 0.3 & 0.20 & & 0.125 & 0.108 \\
\hline 13 & Aspergillus niger & 0.50 & 1.00 & 0.40 & 0.60 & 0.625 & 0.50 & 0.30 & 0.20 & 0.20 & 0.30 & 0.50 & 0.3 & 0.20 & 0.40 & 0.35 & 0.425 \\
\hline 14 & Aspergillus ochraceus & - & - & - & 0.65 & 0.15 & 0.70 & - & - & - & 0.175 & - & - & - & - & - & 0.108 \\
\hline 15 & Aspergillus niveus & 0.30 & - & - & - & .075 & - & - & - & - & - & - & - & 0.20 & 0.40 & 0.15 & 0.075 \\
\hline 16 & Aspergillus terreus & 0.40 & 0.60 & - & - & 0.25 & 0.40 & - & 0.30 & - & 0.175 & - & - & - & - & - & 0.141 \\
\hline 17 & Aspergillus versicolor & 1.10 & 0.80 & 0.10 & & 0.5 & 0.50 & 0.30 & 0.30 & 0.20 & 0.325 & & & 0.10 & 0.20 & 0.075 & 0.30 \\
\hline 18 & Cladosporium cladosporioides & - & - & - & 0.60 & 0.15 & 0.60 & 0.5 & 0.6 & 0.50 & 0.55 & - & - & - & - & - & 0.233 \\
\hline 19 & Cladosporium oxysporum & 0.50 & - & - & 0.50 & 0.125 & 0.50 & 0.4 & 0.4 & 0.30 & 0.4 & 0.80 & 0.70 & - & - & 0.375 & 0.341 \\
\hline 20 & Cladosporium sphaerospermum & 0.30 & 0.20 & 0.60 & - & 0.275 & 0.50 & 0.5 & 0.6 & 0.40 & 0.5 & - & - & - & - & - & 0.258 \\
\hline
\end{tabular}




\begin{tabular}{|c|c|c|c|c|c|c|c|c|c|c|c|c|c|c|c|c|c|}
\hline S.N. & NAME OF FUNGI & July & Aug & Sep & Oct & Total & Nov & Dec & Jan & Feb & Total & Mar & April & May & June & Total & Grand Total \\
\hline 21 & Colletotrichum dematium & - & - & 0.30 & - & 0.075 & - & - & 0.5 & - & 0.125 & - & - & - & - & - & 0.066 \\
\hline 22 & Corynespora cassiicola & 0.30 & 0.20 & 0.30 & 0.20 & 0.25 & 0.20 & - & - & - & 0.05 & - & - & - & - & - & 0.1 \\
\hline 23 & Curvularia borreriae & - & - & - & 0.10 & 0.025 & 0.50 & - & - & - & 0.125 & - & - & - & - & - & 0.05 \\
\hline 24 & Curvularia clavata & 0.20 & 0.50 & 0.80 & & 0.375 & 0.60 & 0.5 & 0.4 & 0.30 & 0.45 & 0.30 & 0.10 & - & 0.20 & 0.15 & 0.325 \\
\hline 25 & Curvularia eragrostidis & - & - & - & - & - & - & - & - & - & - & 0.10 & 0.20 & - & - & 0.075 & 0.025 \\
\hline 26 & Curvularia lunata var. aeria & - & - & - & 0.70 & 0.175 & - & - & - & - & - & - & - & - & - & & 0.058 \\
\hline 27 & Curvularia lunata & 0.30 & 0.50 & 0.30 & & 0.275 & 0.30 & 0.70 & 0.03 & 0.50 & 0.45 & 0.20 & 0.20 & 0.10 & 0.20 & 0.175 & 0.3 \\
\hline 28 & Curvularia ovoidea & - & - & - & - & - & - & 0.20 & 0.10 & - & 0.075 & - & - & - & - & - & 0.025 \\
\hline 29 & Curvularia pallescens & - & - & - & - & - & 0.20 & - & - & - & 0.05 & - & - & - & - & - & 0.166 \\
\hline 30 & Curvularia senegalensis & - & - & - & - & - & - & 0.20 & - & - & 0.05 & - & - & - & - & - & 0.016 \\
\hline 31 & Diplococcium $s p$ & 0.20 & & & 0.30 & 0.125 & 0.40 & 0.70 & 0.30 & & 0.35 & - & - & - & - & - & 0.158 \\
\hline 32 & Drechslera autstraliensis & - & - & - & - & - & - & - & - & - & - & - & - & 0.10 & 0.20 & 0.075 & 0.025 \\
\hline 33 & Drechslera hawaiiensis & - & - & - & 0.20 & 0.05 & 0.40 & 0.30 & - & - & 0.175 & - & - & - & - & - & 0.09 \\
\hline 34 & Epicoccum purpurascens & - & - & 0.20 & 0.10 & 0.075 & 0.30 & 0.20 & - & 0.30 & 0.125 & - & - & - & - & - & 0.066 \\
\hline 35 & Fusarium moniliforme & - & - & - & 0.20 & 0.05 & 0.30 & 0.30 & 0.20 & - & 0.2 & - & - & - & - & - & 0.083 \\
\hline 36 & Fusarium oxysporum & 0.30 & 0.50 & 0.40 & 0.60 & 0.45 & 0.30 & 0.30 & 0.30 & & 0.225 & 0.20 & 0.20 & 0.10 & & 0.125 & 0.266 \\
\hline 37 & Glioclodium viride & - & - & 0.20 & - & 0.05 & - & - & - & - & - & - & - & - & - & - & 0.016 \\
\hline 38 & Macrophomina sp. & - & - & - & 0.50 & 0.125 & 0.10 & 0.20 & - & - & 0.075 & - & - & - & - & - & 0.066 \\
\hline 39 & Monodictys fluctuate & - & - & - & - & - & - & - & - & - & - & - & - & - & 0.30 & 0.075 & 0.025 \\
\hline 40 & Nigrospora oryzae & 0.20 & - & - & 0.30 & 0.125 & 0.30 & 0.20 & 0.50 & 0.30 & 0.325 & & & & 0.30 & 0.075 & 0.175 \\
\hline 41 & Nigrospora sphaerica & 0.10 & - & - & - & 0.025 & - & - & - & - & - & - & - & - & 0.40 & 0.10 & 0.014 \\
\hline 42 & Paecilomyces varioti & - & - & 0.10 & 0.20 & 0.075 & - & - & - & - & - & - & - & - & - & - & 0.025 \\
\hline 43 & Penicillium purpurogenum & - & - & - & 0.10 & 0.025 & - & - & - & - & - & - & - & - & - & - & 0.008 \\
\hline 44 & Penicillium notatum & 0.30 & - & - & - & 0.075 & - & - & 0.60 & 0.30 & 0.225 & - & - & - & - & - & 0.1 \\
\hline 45 & Penicillium rugulosum & - & - & - & - & - & - & - & - & 0.10 & 0.025 & 0.20 & 0.30 & - & - & 0.125 & 0.05 \\
\hline 46 & Periconia sp. & - & - & - & - & - & - & - & - & - & - & - & - & 0.10 & - & 0.025 & 0.008 \\
\hline 47 & Pestalotiopsis glandicola & - & - & - & - & - & - & 0.20 & - & - & 0.05 & - & - & - & - & - & 0.016 \\
\hline 48 & Pithomyces graminicola & - & - & - & - & - & 0.20 & - & - & - & 0.05 & - & - & - & - & - & 0.016 \\
\hline 49 & Trichoderma $s p$ & - & - & - & - & - & 0.20 & 0.30 & 0.20 & - & 0.175 & - & - & - & - & - & 0.058 \\
\hline 50 & Uloclaodium alternariae & - & - & 0.20 & 0.50 & 0.175 & - & 0.20 & - & - & 0.05 & - & - & - & - & - & 0.075 \\
\hline 51 & Mycelia sterila black & 0.20 & 0.10 & - & 0.30 & 0.15 & - & 0.10 & 0.10 & 0.20 & 0.1 & - & 0.20 & - & 0.10 & 0.075 & 0.108 \\
\hline
\end{tabular}


Table.3 Showing Meteorological Data

\begin{tabular}{cccccc}
\hline S.No & Month & \multicolumn{2}{c}{ Temperature } & $\begin{array}{c}\text { Relative } \\
\text { Humidity }\end{array}$ & $\begin{array}{c}\text { Rainfall } \\
{[\mathbf{m m}]}\end{array}$ \\
\hline 1 & Maximum & Minimum & 69.50 & 026.00 \\
2 & July & 31.80 & 24.3 & 60.00 & 031.00 \\
3 & August & 29.10 & 24.4 & 48.50 & 011.60 \\
4 & September & 32.20 & 24.4 & 39.00 & 004.20 \\
5 & October & 30.80 & 19.3 & 35.00 & 008.90 \\
6 & November & 30.20 & 13.8 & 62.00 & 252.20 \\
7 & December & 28.10 & 10.9 & 83.50 & 379.80 \\
8 & January & 26.80 & 11.9 & 86.00 & 156.60 \\
9 & February & 28.90 & 12.3 & 80.00 & 136.40 \\
10 & March & 36.60 & 17.7 & 71.50 & 020.20 \\
11 & April & 39.90 & 23.4 & 60.50 & 000.00 \\
12 & May & 41.10 & 27.9 & 61.00 & 000.00 \\
\hline
\end{tabular}

Table.4 Showing Spearman's Correlation

\begin{tabular}{|c|c|c|c|c|c|c|}
\hline \multicolumn{7}{|c|}{ Correlations } \\
\hline & & & $\begin{array}{l}\text { Total no } \\
\text { colony }\end{array}$ & RH & Temp & Rainfall \\
\hline \multirow[t]{12}{*}{ Spearman's rho } & Total no & Correlation Coefficient & 1.000 & & & \\
\hline & colony & Sig. (2-tailed) & & & & \\
\hline & & $\mathrm{N}$ & 12 & & & \\
\hline & $\mathrm{RH}$ & Correlation Coefficient & $-.680^{*}$ & 1.000 & & \\
\hline & & Sig. (2-tailed) & .015 & & & \\
\hline & & $\mathrm{N}$ & 12 & 12 & & \\
\hline & Temp & Correlation Coefficient & -.382 & -.140 & 1.000 & \\
\hline & & Sig. (2-tailed) & .221 & .665 & & \\
\hline & & $\mathrm{N}$ & 12 & 12 & 12 & \\
\hline & Rainfall & Correlation Coefficient & .247 & $-.676^{*}$ & $.669^{*}$ & 1.000 \\
\hline & & Sig. (2-tailed) & .438 & .016 & .017 & \\
\hline & & $\mathrm{N}$ & 12 & 12 & 12 & 12 \\
\hline
\end{tabular}


Drechslera australiensis, Paecilomyces varioti, Periconia sp. Pestalotiopsis glandicola, Pithomyces graminicola, Glioclodium viride, Tircoderma sp. and Monodicyts fluctuate.

On the contrary some fungal species were showed moderate density, like Chaetomium globosum, Aspergillus niveus, A. terrus, A. ochraceus, A. nidulans, Aspergillus nidulans var. sacristatus, Penicillium rugulosum, $P$. notatum, $A$. radicina, A. citri, $A$. chlamydospora, Curvularia borreriae, Drechslera hawaiiensis, Collectotrichum dematium, Cladosporium cladosporioids, Cladosporium sphaerosperum, Nigrospora sphaerica, Fusarium moniliform, Epicoccum purpurasecns, Uloclodium alteranariae, Corynespora cassicola, Microphomina sp. and Diplococcium. In Rainy season maximum density shown by Alternaria alternate, A.citri. Aspergillus niger.In winter season maximumdensity shown by Aspergillus flavus, Cladosprum cladosporids. C. sphaerospermum, Curvularia clavata, Diplococcium. In Summere the maxium density show by Aspergillus fumigates, Asp.flavus, Cladoserium oxysporum.

The meteorological data was present in the Table No.3 Maximum number of fungal population was recorded during winter season due to favorable temperature $\left(28.46^{\circ} \mathrm{C}\right)$. Moderate number of fungal species during rainy season due to temperature slightly favorable $\left(32.86^{\circ} \mathrm{C}\right)$. Minimum number of fungal population in summer season was due to unfavorable temperature $\left(38.47^{\circ} \mathrm{C}\right)$ Figure No.1.

The influences of Temp, RH and RAF on total numbers of colony (TNC) were analyses statistically through linear correlation and were shown in equation $1,2,3$. The regression with temperature is quite good about 0.709 , while with rainfall is about 0.254 that shows that temperature effect more on the spore of related fungi while effect of rainfall is low as due heavy rainfall flashed out the spore. The effects of time of sampling on the data were also effect as in rainy time than it effect on TNC. The moderate relation of RH on TNC was 0.427.Althrough some author also state the significance of the correlation with humidity on the number of spore (Oliveira 2009).

$\mathrm{TNC}=42.70536( \pm 3.32138)-0.15548( \pm 0.04884)$

Temp, $\mathrm{R}=0.70946, \mathrm{~N}=12 \quad\{1\}$

$\mathrm{TNC}=84.2084( \pm 14.83363)-0.32648( \pm 0.21813)$

$\mathrm{RH}, \mathrm{R}=0.42781, \mathrm{~N}=12 \quad\{2\}$

$\mathrm{TNC}=-8.521( \pm 118.99382)+1.45135( \pm 1.74981)$

$\mathrm{RAF}, \mathrm{R}=0.25371, \mathrm{~N}=12 \quad\{3\}$

The non parametric correlation among total number of colony and all meteorological factor were shown as Spearman's correlation in Table No.4. From table the total number of colony showing positive correlation with rainfall, while negative with temperature and $\mathrm{RH}$.

In conclusion, the analysis of data indicates that concentration of airborne fungi over Barleria prionitis environment is very high and quite variable depending on the climatic conditions. The seasonal climate had positive variations of the influence on occurrence of aeromycoflora. Winter and rainy months registered maximum density of fungal spores due to favorable growth and sporulating conditions for fungi and availability of suitable.

\section{References}

Anderson, A. 1985. Microfungi in beds and their relation to house dust mites. Grana, 24: 55-59. 
Burch, M., Levetin, E. 2002. Effects of meteorological conditions on spore plumes. Int. Arch. Allergy Immunol., 46:107-117.

Troutt, C., Levetin, E. 2001. Correlation of spring spore concentrations and meteorological conditions in Tulsa, Oklahoma. Int. J. Biometeorol., 45: 6474.

Sabariego, S., Díaz De La Guardia, C., Alba, F. 2000. The effect of meteorological factors on the daily variation of airborne fungal spores in Granada (southern Spain). Int. J. Biometeorol., 44: 1-5.

Kasprzyk, E.M. 2006. Work Airborne fungal spores in urban and rural environments in Poland. Aerobiologia, 22: 169-176.

Horner, W.E., Helbling, A., Salvaggio, J.E., Lehrer, S.B. 1995. Fungal allergens. Clin. Microbial. Rev., 8: 161-79.

Rodríguez-Rajo, F., Iglesias, I., Jato, V. 2005. Variation assessment of airborne Alternaria and ladosporium spores at different bioclimatical conditions. Mycol. Res., 109: 497-507a.

Swapna, B., Musale., Vaibhav, A., Jagtap, Minal, S., Patil, K.P., Chittam, Wagh, R.D. 2011. Int. J. Drug Discovery Herbal Res. Earch, 1(1): 20-21.

Purohit, S.S., Vyas, S.P. 2004. Medicinal plants cultivation a scientific approach including processing and financial guidelines. 1stedition, Jodhpur, India. Agrobios, pp. 1-3.

Diksha Khare, K.L., Tiwari. 2015. Studies of Aeromycoflora of Cassia tora L. Int. J. Sci. Res., 4(1): 663-665.

Oliveira, M., Ribeiro, H., Delgado, J.L., Abreu, I. 2009. The effects of meteorological factors on airborne fungal spore concentration in two areas differing in urbanisation level. Int. J. Biometeorol., 53: 61-73.

\section{How to cite this article:}

Diksha Khar and Tiwari, K.L. 2016. Effect of Meteorological Factor on Fluctuation of Aeromycoflora of Barleria prionitis L. Int.J.Curr.Microbiol.App.Sci. 5(3): 459-465.

doi: http://dx.doi.org/10.20546/ijcmas.2016.503.054 